\title{
INTERNATIONAL UNION OF
}

CRYSTALLOGRAPHY

Detailed Fund Accounts

Year ended 31 December 2011 


\section{ADDITIONAL INFORMATION}

Additional Information

Fund accounts summary

General fund account

President's fund account

Journals Fund

International Tables account

Publications and journals development fund account

Research and education fund account

Ewald fund account

Newsletter fund account 
INTERNATIONAL UNION OF CRYSTALLOGRAPHY

\section{ADDITIONAL INFORMATION}

The additional information contained in the detailed fund accounts analysis has been prepared from the accounting records of the Union. While it does not form part of the audited financial statements, it should be read in conjunction with them and the independent auditor's report thereon. 


\section{INTERNATIONAL UNION OF CRYSTALLOGRAPHY}

\section{FUND ACCOUNTS SUMMARY}

Year ended 31 December 2011

General Fund

President's Fund

Journals Fund

International Tables

Publications and Journals Development Fund

Research and Education Fund

Ewald Fund

Newsletter Fund

General Assembly and Congress
Swiss Francs

2011

(Deficit)/

surplus of Decrease in

As at Transfers income over market Fluctuations in exchange Balance at

$\begin{array}{rrrccr}\text { 1 January } & \begin{array}{r}\text { Transfers } \\ \text { between }\end{array} & \begin{array}{c}\text { income over } \\ \text { expenditure }\end{array} & \begin{array}{c}\text { market } \\ \text { value of }\end{array} & \begin{array}{c}\text { Fluctuations in exchange } \\ \text { rates (note 2) }\end{array} & \text { Balance at } \\ \text { fo11 } & \text { funds } & \text { for the year } & \text { investments } & \text { Trading } & \text { Investments }\end{array}$

$(1,756,091)$

109,650

$3,975,590$

$(420,756)$

969,060

947,915

504,628

9,421

319,305

funds

for the year investments

Trading Investments

2011

$4,658,722$
$(297,548)$

$(38,277)$

900,129

$(67,737)$

1,625

$(135,721)$

$(35,392)$

$(60,073)$

$(127,052)$

139,954
$(53,250)$

12,741

(432)

$(27,520)$

2,954

$(6,021)$

$(5,819)$

$(3,140)$

306

$(1,767)$

$(28,698)$

$(53,250)$
$(14,608)$

$(2,108,756)$

70,941

$4,523,199$

$(485,539)$

989,664

956,375

516,096

$(50,346)$

290,486

(28,698)

$(14,608)$

$4,702,120$ 
GENERAL FUND ACCOUNT

\section{Year ended 31 December 2011}

Income

Subscriptions from adhering Bodies

Income from investments

Interest on bank accounts

Amounts charged to the following journals and publications:

Acta Crystallographica

Journal of Applied Crystallography

Journal of Synchrotron Radiation

\section{TOTAL INCOME}

Note

Swiss Francs

2011

2010

153,000

88,162

73,673

1,124

971

153,014

11,169

170,778
17,635

Subscriptions to ICSU and ICSU bodies

Administrative expenses:

General Secretary and Treasurer:

Honorarium to Treasurer

Audit and accountancy charges

Legal and professional fees

Travelling expenses

Bank charges

Executive Secretary's office:

Salaries and expenses

Depreciation of computer and office equipment

Net (income)/expense from book sales

Programming and development costs

Promotion

Meeting of the Executive Committee

Finance Committee expenses

Travel expenses of IUCr Representatives on other bodies

Sponsorship of meetings

IUCr/FIZ agreement

9,614

56,575

7,975

14,860

2,274

289,389

9,385

$(1,106)$

189,812

32,704

72,446

9,617

7,205

7,611

$(15,457)$
TOTAL EXPENDITURE

DEFICIT OF INCOME OVER EXPENDITURE
409,575

14,219

181,818

423,951

11,400

9,544

59,186

7,538

5,279

2,569

305,507

1,659

390,072

391,282

\section{6}

207,539

36,907

63,030

14,864

16,922

4,479

$(18,862)$ 
GENERAL FUND ACCOUNT (continued)

\section{Year ended 31 December 2011}

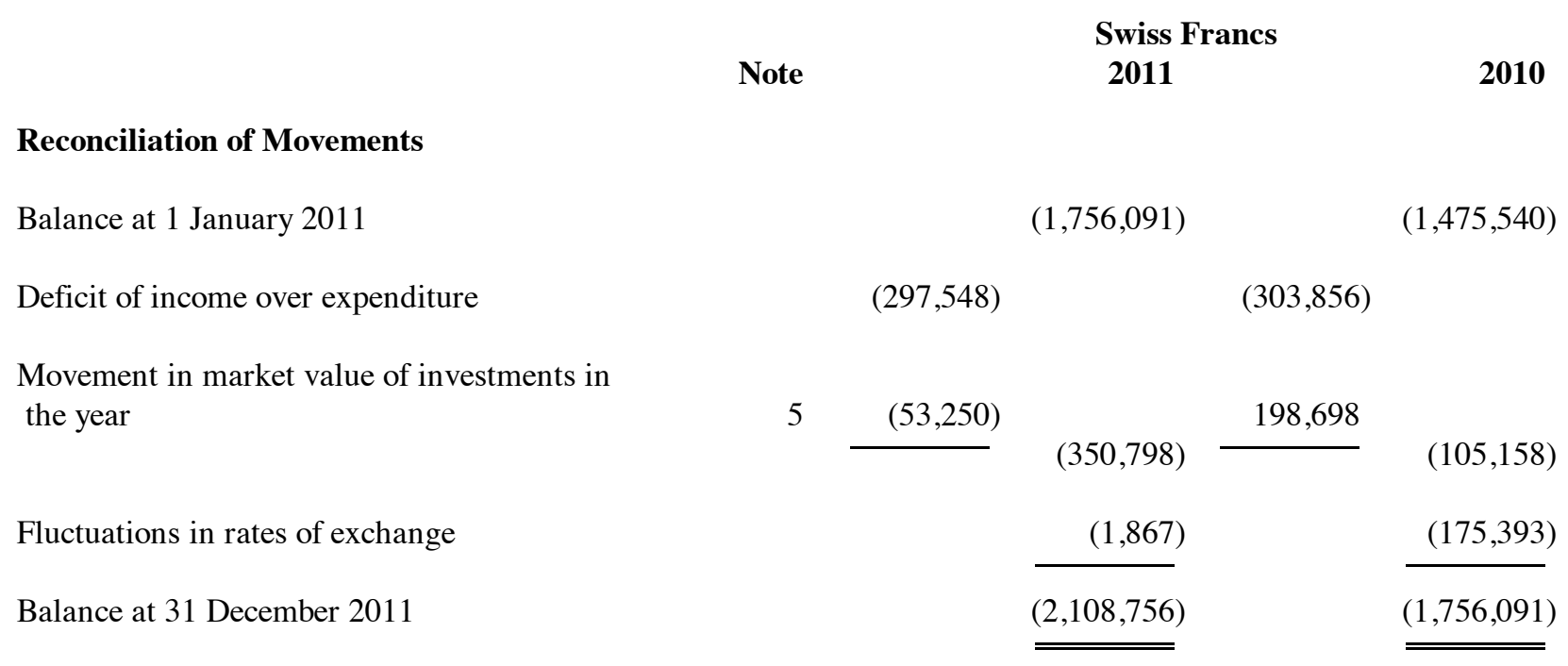


PRESIDENT'S FUND ACCOUNT

Year ended 31 December 2011

\begin{tabular}{|c|c|c|c|}
\hline & Note & $\begin{array}{c}\text { Swiss F } \\
2011\end{array}$ & 2010 \\
\hline $\begin{array}{l}\text { Income } \\
\text { Investment income }\end{array}$ & 7 & - & - \\
\hline $\begin{array}{l}\text { Expenditure } \\
\text { Grants }\end{array}$ & & $(38,277)$ & $(16,738)$ \\
\hline $\begin{array}{l}\text { DEFICIT OF INCOME OVER } \\
\text { EXPENDITURE }\end{array}$ & & $(38,277)$ & $(16,738)$ \\
\hline Reconciliation of movements & & & \\
\hline Balance at 1 January 2011 & & 109,650 & 106,534 \\
\hline $\begin{array}{l}\text { Transfers from other Funds: } \\
\text { Journals fund }\end{array}$ & & - & 25,000 \\
\hline Deficit of income over expenditure & & $(38,277)$ & $(16,738)$ \\
\hline Fluctuations in rates of exchange & & $(432)$ & $(5,146)$ \\
\hline Balance at 31 December 2011 & & 70,941 & 109,650 \\
\hline
\end{tabular}


JOURNALS FUND

Year ended 31 December 2011

Income

Subscriptions \& sale of back numbers and single copies

Royalties and copyright fees

Advertising income

Special issue income

Pay per view and secondary services (net)

Less Publisher's commission on sales

\begin{tabular}{r}
$3,511,497$ \\
32,758 \\
642 \\
16,612 \\
19,763 \\
\hline $3,581,272$ \\
$(405,114)$
\end{tabular}

Swiss Francs

2011

Income from advertisements (net)

Income from copyrights

Income from facility information pages

Recharge for special issue

$\begin{array}{r}4,017,917 \\ 49,575 \\ 4,553 \\ 43,086 \\ 31,325 \\ \hline 4,146,456 \\ (466,500) \\ \hline 3,176,158 \quad \quad \\ \hline\end{array}$

$3,676,956$

3,075

1,759

48,485

$(6,472)$

\section{TOTAL INCOME}

$3,223,005$

Bad debts

Editorial expenses:

Editorial honoraria

Secretarial assistance

Postage, travel and sundries

Technical Editing:

Salaries and expenses

Computer expenses

Subscription administration

Promotion

Depreciation of computer and office equipment

Programming and development

Administration expenses recharged from

General Fund

TOTAL EXPENDITURE

SURPLUS OF INCOME OVER

EXPENDITURE
113,954

$(11,984)$

10,140

291

7,498

122,022

$(14,087)$

22,612

13,554

$(3,170)$

119,899

252,956

2,965

14,928

$1,262,406$

7,620

104,225

98,257

26,177

$1,769,534$

38,888

262,665

$1,954,987$

301,114

170,778

181,818

$2,322,876$

$2,578,830$

900,129 
JOURNALS FUND (continued)

Year ended 31 December 2011

Swiss Francs

2011

2010

Reconciliation of Movements

Balance at 1 January 2011

$3,975,590$

$3,440,171$

Transfers to other Funds:

Publications and Journals Development Fund

$(25,000)$

$(150,000)$

Research and Education Fund

$(100,000)$

$(75,000)$

General Assembly and Congress Fund

-

Newsletter Fund

$(100,000)$

President's Fund

Ewald Fund

$(50,000)$

$(325,000)$

$(410,000)$

Surplus of income over expenditure

900,129

$1,132,016$

Fluctuations in rates of exchange

$(27,520)$

$(186,597)$

Balance at 31 December 2011

$4,523,199$

$3,975,590$ 
INTERNATIONAL TABLES ACCOUNT

\section{Year ended 31 December 2011}

\author{
Income \\ Sales of copies \\ Volume A \\ Volume A1 \\ Volume B \\ Volume C \\ Volume D \\ Volume E \\ Volume F \\ Volume $\mathrm{G}$ \\ Online only complete set \\ Teaching Edition of Volume A
}

Less Publisher's commission on sales

TOTAL INCOME

\section{Expenditure}

Publication expenses:

Production Volume A

Production Volume A1

Production Volume B

Production Volume C

Production Volume D

Production Volume E

Production Volume F

Production Volume G

Production Teaching Edition Volume A

Editorial expenses:

Editorial honoraria

Technical Editing

Programming and development

Promotion

TOTAL EXPENDITURE

(DEFICIT)/ SURPLUS OF INCOME OVER EXPENDITURE

\section{Swiss Francs}

2011

2010

\begin{tabular}{cr}
20,658 & 30,830 \\
$(13,925)$ & 63,250 \\
9,270 & 15,826 \\
9,896 & 14,437 \\
6,948 & 10,928 \\
9,728 & 26,935 \\
7,633 & 14,194 \\
6,152 & 7,540 \\
66,803 & 90,072 \\
1,088 & 3,613 \\
\hline 124,251 & 277,625 \\
$(31,338)$ & $(68,842)$ \\
\hline
\end{tabular}

92,913

208,783

10,587

$(2,464)$

2,157

3,209

9,913

557

1,985

11,239

-

7,539

12,877

4,344

3,882

31,275

1,979

2,774

$(13,517)$

3,649

37,183

54,802

9,749

79,378

76,863

88,442

89,127

21,139

18,454

16,352

183,522

160,650

$(67,737)$

25,261 
INTERNATIONAL TABLES ACCOUNT (continued)

Year ended 31 December 2011

$\begin{array}{cc}\text { Swiss } & \text { Swiss } \\ \text { Francs } & \text { Francs }\end{array}$

20112010

Reconciliation:

Balance at 1 January 2011

$(420,756)$

$(465,765)$

(Deficit)/surplus of income over expenditure

$(67,737)$

25,261

Fluctuations in rates of exchange

2,954

19,748

Balance at 31 December 2011

$(485,539)$

$(420,756)$ 
PUBLICATIONS AND JOURNALS DEVELOPMENT FUND ACCOUNT

Year ended 31 December 2011

\begin{tabular}{|c|c|c|c|c|}
\hline & & $\begin{array}{c}\text { Swiss I } \\
2011\end{array}$ & ancs & 2010 \\
\hline Income & & & & \\
\hline STAR/CIF income & & 15,087 & & 13,113 \\
\hline & & 15,087 & & 13,113 \\
\hline Expenditure & & & & \\
\hline Computer Expenses: & & & & \\
\hline Programming and development & 471,150 & & 529,792 & \\
\hline Recharged to other funds & $(471,150)$ & & $(529,792)$ & \\
\hline $\begin{array}{l}\text { Electronic publishing committee/section } \\
\text { editors meeting }\end{array}$ & 14.613 & & 3576 & \\
\hline Special issue (surplus)/deficit from other funds & $(7,520)$ & & $(20,893)$ & \\
\hline Archiving & 399 & & - & \\
\hline STAR/CIF & - & & 8,667 & \\
\hline Promotion & 147,313 & & 166,249 & \\
\hline Promotion recharged to other funds & $(147,313)$ & & $(166,249)$ & \\
\hline Web input & 515 & & 656 & \\
\hline Journal subscription subsidies & 4,521 & & 4,397 & \\
\hline Depreciation & 934 & & 934 & \\
\hline TOTAL EXPENDITURE & & 13,462 & & $(2,663)$ \\
\hline SURPLUS OF INCOME OVER EXPENDITURE & & 1,625 & & 15,776 \\
\hline Reconciliation of Movements & & & & \\
\hline Balance at 1 January 2011 & & 969,060 & & 923,768 \\
\hline Transfers from other Funds: & & & & \\
\hline Journals fund & & 25,000 & & 75,000 \\
\hline Surplus of income over expenditure & & 1,625 & & 15,776 \\
\hline Fluctuations in rates of exchange & & $(6,021)$ & & $(45,484)$ \\
\hline Balance at 31 December 2011 & & 989,664 & & 969,060 \\
\hline
\end{tabular}


RESEARCH AND EDUCATION FUND ACCOUNT

Year ended 31 December 2011

\begin{tabular}{|c|c|c|c|c|c|}
\hline \multirow{2}{*}{ Income } & Note & Swiss Francs & $\begin{array}{c}\text { Swiss I } \\
2011\end{array}$ & & 2010 \\
\hline & & & & & \\
\hline Investment income & 7 & & - & & - \\
\hline Expenditure & & & & & \\
\hline Young Scientists' Support & & 123,179 & & 126,513 & \\
\hline Visiting professorship programme & & 12,542 & & 5,048 & \\
\hline Africa PHD Support & & & & 10,907 & \\
\hline TOTAL EXPENDITURE & & & 135,721 & & 142,468 \\
\hline DEFICIT OF INCOME OVER & & & & & \\
\hline EXPENDITURE & & & $(135,721)$ & & $(142,468)$ \\
\hline Reconciliation of movements & & & & & \\
\hline Balance at 1 January 2011 & & & 947,915 & & 974,874 \\
\hline Transfers from other Funds: & & & & & \\
\hline Journals fund & & & 150,000 & & 160,000 \\
\hline Deficit of income over expenditure & & & $(135,721)$ & & $(142,468)$ \\
\hline Fluctuations in rates of exchange & & & $(5,819)$ & & $(44,491)$ \\
\hline Balance at 31 December 2011 & & & 956,375 & & 947,915 \\
\hline
\end{tabular}


EWALD FUND ACCOUNT

Year ended 31 December 2011

\begin{tabular}{|c|c|c|c|}
\hline & Note & $\begin{array}{c}\text { Swiss F } \\
2011\end{array}$ & 2010 \\
\hline \multicolumn{4}{|l|}{ Income } \\
\hline Investment income & 7 & - & - \\
\hline \multicolumn{4}{|l|}{ Expenditure } \\
\hline Ewald prize & & 35,392 & - \\
\hline DEFICIT OF INCOME OVER & & & \\
\hline EXPENDITURE & & $(35,392)$ & - \\
\hline \multicolumn{4}{|l|}{ Reconciliation of movements } \\
\hline Balance at 1 January 2011 & & 504,628 & 528,313 \\
\hline Transfer from other Funds: & & & \\
\hline Journals fund & & 50,000 & - \\
\hline Deficit of income over expenditure & & $(35,392)$ & - \\
\hline Fluctuations in rates of exchange & & $(3,140)$ & $(23,685)$ \\
\hline Balance at 31 December 2011 & & 516,096 & 504,628 \\
\hline
\end{tabular}


NEWSLETTER FUND ACCOUNT

Year ended 31 December 2011

Swiss Francs

2011

2010

Income

Income from advertisements

118,900

147,176

\section{Expenditure}

Editorial Honoraria

2,882

3,206

Editorial Expenses

89,477

121,501

Newsletter printing and distribution

86,614

113,779

TOTAL EXPENDITURE

178,973

238,486

DEFICIT OF INCOME OVER EXPENDITURE

$(60,073)$

$(91,310)$

Reconciliation of Movements

Balance at 1 January 2011

9,421

51,173

Transfers from other Funds:

Journals fund

50,000

Deficit of income over expenditure

$(60,073)$

$(91,310)$

Fluctuation in rates of exchange

306

Balance at 31 December 2011

$(50,346)$

9,421 
GENERAL ASSEMBLY AND CONGRESS FUND

Year ended 31 December 2011

Swiss Francs

2011

2010

\section{Income}

Investment income

\section{Expenditure}

Programme committee meeting

Congress expenses

Travel expenses
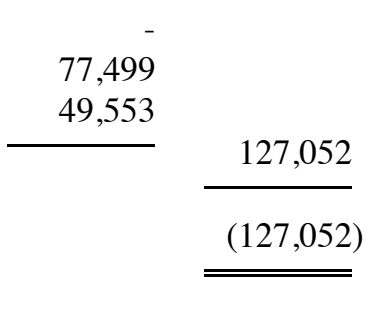

3,134

10,186

DEFICIT OF INCOME OVER EXPENDITURE

319,305

61,175

$(61,175)$

\section{Reconciliation of movements}

Balance at 1 January 2011

295,468

Transfers from other Funds:

Journals fund

\begin{tabular}{|c|c|c|c|}
\hline 100,000 & & 100,000 & \\
\hline & 100,000 & & 100,000 \\
\hline & $(127,052)$ & & $(61,175)$ \\
\hline & $(1,767)$ & & $(14,987)$ \\
\hline & 290,486 & & 319,305 \\
\hline
\end{tabular}

